\title{
Realización de destrezas de vía aérea en el primer año de residencia en el Centro Médico ABC: un análisis retrospectivo de cuatro generaciones
}

\author{
Airway skills performance by first year residents at the ABC \\ Medical Center: a retrospective analysis of four generations
}

\author{
Juan Sebastián Espino-Núñez, ${ }^{*}$ Atzin Suá Ruiz-Hernández, ${ }^{\ddagger}$ Daniela Beatriz Vázquez-Hernández, ${ }^{\ddagger}$ \\ Rodrigo Rubio-Martínez,§ Marco Antonio Chávez-Ramírez
}

Citar como: Espino-Núñez JS, Ruiz-Hernández AS, Vázquez-Hernández DB, Rubio-Martínez R, Chávez-Ramírez MA. Realización de destrezas de vía aérea en el primer año de residencia en el Centro Médico ABC: un análisis retrospectivo de cuatro generaciones. An Med ABC. 2021; 66 (3): 190-194. https://dx.doi.org/10.35366/101666

\section{RESUMEN}

Introducción: El desarrollo de destrezas en la vía aérea es importante en la preparación de un anestesiólogo. En el Centro Médico $\mathrm{ABC}$ los residentes de anestesiología llevan una bitácora del número de destrezas realizadas durante su entrenamiento. Objetivo: Determinar el número de destrezas de la vía aérea realizadas durante el primer año de residencia de cuatro generaciones de residentes de anestesiología del Centro Médico ABC. Material y métodos: Se incluyeron los residentes de primer año durante el periodo de 2016-2019. Las destrezas de la vía aérea estudiadas fueron: laringoscopia directa, videolaringoscopia, fibroscopia y la colocación de mascarilla laríngea de cualquier generación y marca. Los datos se obtuvieron de los reportes de procedimiento mensuales durante el primer año de residencia entregados al Departamento de Anestesiología del Centro Médico ABC. Resultados: Veintiún residentes fueron incluidos en el estudio. En su primer año de residencia participaron en un total de 11,140 cirugías, de las cuales en 8,464 realizaron alguna destreza de la vía aérea. La intubación orotraqueal por laringoscopia directa fue la más frecuente, con un promedio de $262.66 \pm 58.84$ anuales

\section{ABSTRACT}

Introduction: The development of airway skills is important for the training of an anesthesiologist. At the ABC Medical Center, residents of anesthesiology present a logbook with the number of skills performed during their training. Objective: To determine the number of airway skills performed during the first year of residency for four generations of anesthesiology residents at the $A B C$ Medical Center. Material y methods: First-year residents during the 2016-2019 period were included. The airway skills studied were direct laryngoscopy, video laryngoscopy, fibroscopy and the placement of a laryngeal mask of any generation and brand. The data was obtained from the monthly procedure reports delivered to the Department of Anesthesiology of the ABC Medical Center. Results: Twentyone residents were included in the study. In their first year of residence there were a total of 11,140 surgeries. In 8,464 some airway skill was performed. Orotracheal intubation by direct laryngoscopy was the most frequent, with an average of $262.66 \pm$ 58.84 per year, $33.85 \pm 8.32$ by videolaryngoscopy and $3.23 \pm 2.68$ were performed with fibroscopy. Finally, they placed laryngeal masks with an average of $107.10 \pm 18.05$ per year. Conclusions:
* Anestesiología. Profesor de Cátedra de la Escuela de Medicina del Tecnológico de Monterrey.

‡ Médico residente de Anestesiología.

$\S$ Anestesiología. Profesor del Curso de Anestesiología, Universidad Nacional Autónoma de México.

ๆ Anestesiología. Profesor Titular del Curso de Anestesiología, Universidad Nacional Autónoma de México.
Centro Médico ABC. México.

Correspondencia:

Juan Sebastián Espino-Núñez

E-mail: sebastian.espino.nunez@tec.mx

Recibido: 21/03/2021. Aceptado: 17/08/2021. 
por residente, por videolaringoscopia fue de $33.85 \pm 8.32$ y con fibroscopia se realizó un promedio de $3.23 \pm 2.68$. Por último, colocaron mascarillas laríngeas con un promedio de $107.10 \pm 18.05$ anuales. Conclusiones: En su primer año de entrenamiento, los residentes de anestesiología del Centro Médico $\mathrm{ABC}$ realizan en promedio 408 destrezas de la vía aérea.

Palabras clave: Intubación orotraqueal, curva de aprendizaje, entrenamiento en vía aérea, anestesiología.

Nivel de evidencia: IV

\section{INTRODUCCIÓN}

El desarrollo de destrezas en el manejo de la vía aérea es parte medular del entrenamiento de un anestesiólogo. ${ }^{1,2}$ Para este desarrollo es necesaria la realización de las destrezas en un número de ocasiones suficiente para disminuir la posibilidad de fallo en una tarea tan crítica. ${ }^{3}$ El manejo adecuado de la vía aérea es un factor importante que contribuye a disminuir la morbilidad y mortalidad asociada a la anestesia.

El desarrollo de competencia en el manejo de la vía aérea requiere el dominio de una amplia gama de habilidades, desde la ventilación con mascarilla facial hasta la intubación con fibra óptica. Esto incluye no sólo el conocimiento técnico de diversos dispositivos con distinta complejidad, sino también la adquisición del juicio clínico y la experiencia para usarlos de manera adecuada. ${ }^{4,5}$ El Proyecto Nacional de Auditoria del Colegio Real de Anestesiólogos en el Reino Unido (NAP4) evaluó las complicaciones mayores asociadas a la manipulación de la vía aérea. El reporte en 2011 concluyó que la falta de juicio y el entrenamiento inadecuado eran el segundo y tercer factor causal y contribuyente más frecuente de complicaciones, después de los factores relacionados al paciente. ${ }^{6}$

En el Centro Médico ABC (CMABC) la residencia de anestesiología cuenta con una historia de 31 años formando especialistas. Cada año, entre tres y seis residentes son admitidos para realizar su entrenamiento en este Centro. La residencia consiste en tres años de práctica dentro de la sede, además de rotaciones externas y el servicio social de especialidad. Durante este tiempo se tiene acceso a escenarios clínicos de todas las especialidades médico-quirúrgicas y sus respectivos desafíos en el manejo de la vía aérea siempre supervisados por un adscrito de anestesiología con certificación vigente. El CMABC cuenta con una amplia variedad de dispositivos avanzados para la vía aérea que incluyen laringoscopios, videolarin-
During their first year of training, anesthesiology residents from ABC Medical Center performed on average 408 airway skills.

Keywords: Orotracheal intubation, learning curve, airway training, anesthesiology.

Level of evidence: $I V$

goscopios, dispositivos supraglóticos, fibroscopios, así como otros aditamentos disponibles en el carro de vía aérea difícil, a los cuales los residentes tienen acceso durante su formación.

En este estudio observacional, descriptivo y retrospectivo se documentó el número de destrezas realizadas por los residentes en el manejo de la vía aérea durante su primer año de entrenamiento.

El objetivo es determinar el número de destrezas de la vía aérea realizadas por los residentes de anestesiología durante el primer año de entrenamiento.

\section{MATERIAL Y MÉTODOS}

El presente estudio es un reporte preliminar del protocolo presentado ante el Comité de Investigación y de Ética en Investigación del CMABC aprobado con el folio ABC-20-100.

\section{Población de estudio}

Se reunieron consecutivamente de forma retrolectiva los reportes mensuales presentados por todos los residentes de primer año en el CMABC, entre marzo de 2016 y febrero de 2019.

Los datos se obtuvieron de los reportes de procedimiento mensuales durante el primer año de residencia entregados al Departamento de Anestesiología del CMABC. Las destrezas recopiladas fueron: intubación con laringoscopia directa, intubación con videolaringoscopio tipo C-MAC ${ }^{\circledR}$, colocación de mascarilla laríngea e intubación con fibroscopio. En el registro mensual los residentes sólo incluyen el número de destrezas. No se incluyen complicaciones, tasa de éxito $u$ otros desenlaces que son también relevantes para evaluar el entrenamiento.

Se utilizó la prueba de normalidad de ShapiroWilk, posteriormente se utilizaron medidas de tendencia central: media o mediana y dispersión: desviación estándar o rango intercuartil dependiendo del 
resultado de la prueba de normalidad. Se utilizó el software Prism (versión 6.0) para el análisis. Se consideró significación estadística $\mathrm{p}<0.05$.

\section{RESULTADOS}

La población de estudio estuvo constituida por 252 reportes mensuales correspondientes a los primeros 12 meses de 21 residentes de anestesiología. De ellos, 14 fueron mujeres (66.6\%). Cinco residentes corresponden al periodo $2016-2017$ y asistieron a 2,793 cirugías; seis residentes corresponden al periodo 20172018 y asistieron a 3,365 cirugías; cuatro residentes en el periodo 2018-2019 asistieron 1,946 cirugías; y seis residentes del periodo 2019-2020 asistieron a 3,036 cirugías. Un total de 11,140 cirugías fueron asistidas por residentes de primer año en el periodo de marzo de 2016 a febrero de 2020.

El total de intubaciones por laringoscopia directa por residentes de primer año fue de 5,516. El promedio de laringoscopias directas en el mes y el promedio acumulado se muestra en la Tabla 1.

En cuanto a la intubación mediante videolaringoscopia tipo C-MAC ${ }^{\circledR}$, se realizaron 711 procedimientos por residentes de primer año. El promedio de videolaringoscopias por mes y el promedio acumulado se muestra en la Tabla 2.

En cuanto a la colocación de mascarillas laríngeas, se realizaron 2,169 por residentes de primer año. El promedio de mascarillas laríngeas colocadas por mes y el promedio acumulado se muestra en la Tabla 3.

Tabla 1: Laringoscopias realizadas por mes.

\begin{tabular}{lcc}
\hline & Promedio por mes & Promedio acumulado \\
\hline Marzo & $21.76 \pm 14.73$ & $21.76 \pm 14.73$ \\
Abril & $22.09 \pm 8.55$ & $43.85 \pm 11.90$ \\
Mayo & $22.87 \pm 8.48$ & $66.71 \pm 10.67$ \\
Junio & $24.90 \pm 95.0$ & $91.61 \pm 10.49$ \\
Julio & $19.90 \pm 6.16$ & $111.52 \pm 9.78$ \\
Agosto & $24.23 \pm 12.28$ & $135.76 \pm 10.24$ \\
Septiembre & $24.71 \pm 11.01$ & $160.47 \pm 10.34$ \\
Octubre & $19.51 \pm 10.44$ & $180.00 \pm 10.37$ \\
Noviembre & $21.70 \pm 9.96$ & $201.70 \pm 10.30$ \\
Diciembre & $21.00 \pm 6.97$ & $222.70 \pm 10.03$ \\
Enero & $22.75 \pm 11.68$ & $245.45 \pm 10.16$ \\
Febrero & $21.33 \pm 11.44$ & $266.78 \pm 10.26$ \\
\hline
\end{tabular}

Datos cualitativos mostrados como media \pm desviación estándar.
Tabla 2: Videolaringoscopias realizadas por mes.

\begin{tabular}{lcc}
\hline & Promedio por mes & Promedio acumulado \\
\hline Marzo & $2.55(0.5-3)^{\star}$ & $2.55(0.5-3)^{\star}$ \\
Abril & $1.80 \pm 1.47$ & $4.49 \pm 2.02$ \\
Mayo & $2.71(1-4)^{\star}$ & $7.29 \pm 2.17$ \\
Junio & $2.33(0-4.4)^{*}$ & $9.49 \pm 2.14$ \\
Julio & $2.1 \pm 2.02$ & $11.64 \pm 2.11$ \\
Agosto & $2.00 \pm 2.30$ & $13.74 \pm 2.22$ \\
Septiembre & $3.42 \pm 2.48$ & $17.32 \pm 2.22$ \\
Octubre & $3.28(1-4)^{*}$ & $20.77 \pm 2.33$ \\
Noviembre & $3.23 \pm 2.40$ & $24.24 \pm 2.34$ \\
Diciembre & $2.71 \pm 2.65$ & $26.96 \pm 2.37$ \\
Enero & $3.85 \pm 2.65$ & $31.01 \pm 2.42$ \\
Febrero & $3.95 \pm 2.60$ & $34.74 \pm 2.45$ \\
\hline Datos cualitativos mostrados como media \pm desviación estándar. \\
* Datos cuantitativos con distribución no normal, se muestra como mediana \\
(rangos intercuartil 25-75).
\end{tabular}

En cuanto a la intubación mediante fibroscopio, se realizaron 68 procedimientos por residentes de primer año. Hubo tres residentes que no realizaron ninguna intubación con fibroscopio en su primer año y tres que realizaron más de cinco.

\section{DISCUSIÓN}

En el CMABC la formación de anestesiólogos tiene una historia de tres décadas y el número de destrezas de la vía aérea que realizan los residentes no había sido reportado. El entrenamiento de habilidades técnicas requiere de repetición y práctica deliberada. ${ }^{7}$

Específicamente en el manejo de la vía aérea, curvas de aprendizaje han sido descritas para diversos procedimientos. ${ }^{3,8,9}$ Los residentes del CMABC, por ejemplo, al terminar su tercer mes de residencia con un promedio de 66.71 laringoscopias directas ya completaron la curva de aprendizaje descrita. ${ }^{10}$ Para la videolaringoscopia, cada dispositivo disponible tiene diferentes estudios describiendo las curvas de aprendizaje. En el CMABC el videolaringoscopio más utilizado es el C-MAC ${ }^{\circledR}$ con su hoja hiperangulada y no hay información disponible sobre el número de intentos necesarios para lograr un éxito mayor al $90 \%$ en el primer intento.

Las descripciones de curvas de aprendizaje pueden ser de utilidad para el desarrollo de una habilidad dentro del currículum del programa de postgrado. El Consejo de Acreditación para Educación Médica de Postgrado en los Estados Unidos, por ejemplo, 
tiene un mínimo de casos en los que el residente debe participar para poder certificarse ${ }^{11}$ sin embargo, no define un número de habilidades técnicas específicas a realizarse para alcanzar la certificación.

El establecimiento de una bitácora de procedimientos es un primer paso para la documentación de competencias, pero no puede interpretarse como proficiencia sin un contexto más amplio de las complicaciones, número de intentos, etcétera. ${ }^{12} \mathrm{La}$ complementación de una bitácora de procedimientos con un portafolio de evidencia con reflexión de las experiencias ayudaría a completar el ciclo de aprendizaje. ${ }^{13-15}$

La transformación de los postgrados en medicina hacia una educación basada en competencias es la promesa de mayor flexibilidad y la apropiación de la responsabilidad del residente en su propio aprendizaje. ${ }^{16}$ La educación basada en competencias está aún en una etapa de desarrollo de la evidencia que la sustente, pero en áreas como la adquisición de habilidades técnicas existe mayor consenso. ${ }^{17,18}$

El presente estudio tiene limitaciones comenzando por el diseño retrospectivo. Los reportes son obligatorios y son generados por el mismo residente, pero no son usados para su evaluación, por lo que el incentivo de llenarlos correctamente es de beneficio para el residente que lleva un registro de sus casos. Por el diseño de los reportes que se solicitan, no se registra si existió alguna complicación, si el número de intentos fue mayor a uno, si se usó algún aditamento especial como guías o introductores, etcétera. Otra li-

Tabla 3: Colocación de mascarillas laríngeas realizadas por mes.

\begin{tabular}{lcc}
\hline & Promedio por mes & Promedio acumulado \\
\hline Marzo & $8.20 \pm 5.10$ & $8.20 \pm 5.10$ \\
Abril & $10.61(5.5-13.5)^{\star}$ & $18.81 \pm 8.28$ \\
Mayo & $9.85(5.5-12)^{*}$ & $28.67 \pm 7.83$ \\
Junio & $9.85 \pm 5.22$ & $38.52 \pm 7.26$ \\
Julio & $7.19 \pm 4.14$ & $45.72 \pm 7.60$ \\
Agosto & $9.00 \pm 5.33$ & $55.16 \pm 6.80$ \\
Septiembre & $8.62 \pm 4.32$ & $64.22 \pm 6.27$ \\
Octubre & $8.80(6.4)$ & $73.02 \pm 6.27$ \\
Noviembre & $7.95 \pm 4.73$ & $81.36 \pm 6.12$ \\
Diciembre & $6.9 \pm 3.85$ & $88.30 \pm 5.95$ \\
Enero & $8.15 \pm 4.53$ & $94.41 \pm 5.83$ \\
Febrero & $10.65 \pm 6.74$ & $107.10 \pm 5.92$ \\
\hline Datos cualitativos mostrados como media \pm desviación estándar. \\
* Datos cuantitativos con distribución no normal, se muestra como mediana \\
(rangos intercuartil 25-75).
\end{tabular}

mitación es la falta de especificación de los diferentes dispositivos de vía aérea utilizados: existen muchos modelos de videolaringoscopios y sólo el C-MAC ${ }^{\circledR}$ se reporta de forma consistente; o diferentes modelos de mascarillas laríngeas que no son especificados. Es por ello que los resultados deben interpretarse con base en estas limitaciones.

\section{CONCLUSIÓN}

Los residentes de anestesiología del CMABC de las generaciones 2016-2019 realizaron en promedio 408 destrezas de la vía aérea durante su primer año de entrenamiento. La más común fue la laringoscopia directa y completaron de forma temprana la curva de aprendizaje descrita para esta técnica.

\section{REFERENCIAS}

1. Saracoglu KT, Saracoglu A. Airway management education: An update. It is high time to realize professional airway training as an art. Acta Anaesthesiol Belg. 2017; 68 (4): 175186.

2. Baker PA, Weller JM, Greenland KB, Riley RH, Merry AF. Education in airway management. Anaesthesia. 2011; 66 Suppl 2: 101-111.

3. Buis ML, Maissan IM, Hoeks SE, Klimek M, Stolker RJ. Defining the learning curve for endotracheal intubation using direct laryngoscopy: a systematic review. Resuscitation. 2016; 99: 63-71.

4. Ericsson KA. Deliberate practice and the acquisition and maintenance of expert performance in medicine and related domains. Acad Med. 2003; 79 (10): S70-S81.

5. Hastings RH, Rickard TC. Deliberate practice for achieving and maintaining expertise in anesthesiology. Anesth Analg. $2015 ; 120$ (2): 449-459.

6. Cook TM, Woodall N, Frerk C; Fourth National Audit Project. Major complications of airway management in the United Kingdom. e London: The Royal College of Anaesthetists; 2011. pp. 143-155.

7. Ericsson KA, Krampe RT, Tesch-Romer C. The role of deliberate practice in the acquisition of expert performance. Psychol Rev. 1993; 100 (3): 363-406.

8. de Oliveira Filho GR. The construction of learning curves for basic skills in anesthetic procedures: an application for the cumulative sum method. Anesth Analg. 2002; 95 (2): 411-416.

9. Komatsu R, Kasuya Y, Yogo H, Sessler DI, Mascha E, Yang $\mathrm{D}$ et al. Learning curves for bag-and-mask ventilation and orotracheal intubation: an application of the cumulative sum method. Anesthesiology. 2010; 112 (6): 1525-1531.

10. Konrad C, Schüpfer G, Wietlisbach M, Gerber H. Learning manual skills in anesthesiology: is there a recommended number of cases for anesthetic procedures? Anesth Analg. 1998; 86 (3): 635-639.

11. Yamamoto S, Tanaka P, Madsen MV, Macario A. Analysis of resident case logs in an anesthesiology residency program. A A Case Rep. 2016; 6 (8): 257-262.

12. O'Shaughnessy S, Skerritt C, Fitzgerald C, Irwin R, Walsh F. First year specialist anaesthesia training in Ireland: a logbook analysis. Int J High Educ. 2017; 6 (6): 106. 
An Med ABC. 2021; 66 (3): 190-194

13. Fraser AB, Stodel EJ, Chaput AJ. Curriculum reform for residency training: competence, change, and opportunities for leadership. Can J Anesth. 2016; 63 (7): 875-884.

14. Chiu M, Tarshis J, Antoniou A, Bosma TL, Burjorjee JE, Cowie N et al. Simulation-based assessment of anesthesiology residents' competence: development and implementation of the Canadian National Anesthesiology Simulation Curriculum (CanNASC). Can J Anesth. 2016; 63 (12): 1357-1363.

15. Shorten GD, De Robertis E, Goldik Z, Kietaibl S, NiemiMurola L, Sabelnikovs O. European Section/Board of Anaesthesiology/European Society of Anaesthesiology consensus statement on competency-based education and training in anaesthesiology. Eur J Anaesthesiol. 2020; 37 (6): 421-434.

16. Holmboe ES, Sherbino J, Englander R, Snell L, Frank JR. A call to action: the controversy of and rationale for competencybased medical education. Med Teach. 2017; 39 (6): 574-581.

17. Weller JM, Naik VN, San Diego RJ. Systematic review and narrative synthesis of competency-based medical education in anaesthesia. Br J Anaesth. 2020; 124 (6): 748-760.

18. Vinagre R, Tanaka P, Tardelli MA. Competency-based anesthesiology teaching: comparison of programs in Brazil, Canada and the United States. Braz J Anesthesiol. 2021; 71 (2): $162-170$. 